\title{
A framework to start the debate on neonatal screening policies in the EU: an Expert Opinion Document
}

\author{
Martina C Cornel ${ }^{\star, 1}$, Tessel Rigter ${ }^{1}$, Stephanie S Weinreich ${ }^{1}$, Peter Burgard ${ }^{2}$, Georg F Hoffmann ${ }^{2}$, \\ Martin Lindner ${ }^{2}$, J Gerard Loeber ${ }^{3}$, Kathrin Rupp ${ }^{2}$, Domenica Taruscio ${ }^{4}$ and Luciano Vittozzi ${ }^{4}$
}

The European Union (EU) Council Recommendation on rare diseases urged the member states to implement national and EU collaborative actions to improve the health care of rare disease patients. Following this recommendation, the European Commission launched a tender on newborn screening (NBS) to report on current practices of laboratory testing, form a network of experts and provide guidance on how to further implement NBS screening in a responsible way, the latter of which was provided in an Expert Opinion document. After consultation of experts from EU member states, (potential) candidate member states and European Free Trade Association countries, in a consensus meeting in June 2011, 70 expert opinions were finalized. They included the need to develop case definitions for all disorders screened for to facilitate assessment and international outcome studies. Decision whether a screening program should be performed can be based on screening criteria updated from the traditional Wilson and Jungner (1968) criteria, relating to disease, treatment, test and cost. The interest of the child should be central in the assessment of pros and cons. A European NBS body should assess evidence on (new) screening candidate disorders. For rare conditions, best level evidence should be used. The health system should ensure treatment to cases diagnosed by screening, controlled and revised by follow-up outcome studies. Screening methodology should aim to avoid unintended findings, such as mild forms and carrier status information, as much as possible. Activities to improve NBS in Europe, such as training and scientific evaluation, could benefit from collaboration at EU level and beyond.

European Journal of Human Genetics (2014) 22, 12-17; doi:10.1038/ejhg.2013.90; published online 8 May 2013

The European Union (EU) Council Recommendation ${ }^{1}$ on Rare Diseases (9 June 2009) ${ }^{2}$ identified rare diseases (ie, a life-threatening or chronically debilitating condition affecting not more than five in 10000 persons in the community) as a public health concern and highlighted the need for public health actions, promoting the development of research on rare disorders and the improvement of the health care of rare disease patients. Following this recommendation, the European Commission launched a tender on neonatal screening (= newborn screening, NBS) in July 2009 (http:// ec.europa.eu/eahc/health/tenders_H09C2.html) in order to (1) report on the practices of neonatal screening for rare disorders implemented in all the member states, including number of centers, estimate the number of infants screened and the number of disorders included in the NBS, as well as reasons for the selection of these disorders, (2) to identify types of medical management and follow-up implemented in the member states, (3) to establish a network of experts analyzing the information and formulating a final opinion containing recommendations on best practices, and recommending a core panel of NBS conditions that could be included in all MS practices, and (4) to develop a decision-making matrix that could be used by member states' programs to systematically expand (or contract) screening mandates.

The focus of the tender activities was on NBS by using laboratory testing techniques (blood spot screening). All reports are available on the internet (http://www.iss.it/cnmr/prog/cont.php?id=1621\&lang=1\&tipo=64).
To get some insight into the current practices (points 1 and 2 above), an online survey was compiled and filled out by EU member states, (potential) member states and European Free Trade Association countries - in total 40 countries. Apart from the final report, available on the internet, the current practices are summarized in two journal articles: the first publication addresses the steps in screening programmes from blood spot to screening result ${ }^{3}$ and the second publication addresses the steps from screening laboratory results to treatment, follow-up and quality assurance. ${ }^{4}$

As a third part of the activity and work methodology requested by the tender specifications, a European Union Network of Experts on Newborn Screening (EUNENBS) had to be constituted. Criteria for the inclusion of experts in EUNENBS (http://www.iss.it/cnmr/prog/ cont.php?id=1621\&lang=1\&tipo=64) include that all member states' authorities should be represented in the network. Each countries' competent authorities were invited to identify their experts to represent the country at the workshops in 2010 and 2011. Further experts represent European professional and scientific organizations involved in NBS, the representative of the US Secretary's Advisory Committee on Heritable Disorders in Newborns and Children, additional fields of expertise (eg, ethics) and patient organizations. The list of EUNENBS members is available as Appendix 1 of the Expert Opinion document (http://www.iss.it/cnmr/prog/cont. php?id=1621\&lang=1\&tipo=64). Most EUNENBS members have a background in health policy making, health technology assessment

${ }^{1}$ Clinical Genetics and EMGO Institute for Health and Care Research, VU University Medical Centre, Amsterdam, The Netherlands; ${ }^{2}$ Centre for Paediatric and Adolescent Medicine, University of Heidelberg, Heidelberg, Germany; ${ }^{3}$ Laboratory for Infectious Diseases and Perinatal Screening, National Institute for Public Health, Bilthoven, The Netherlands; ${ }^{4}$ National Centre for Rare Diseases, Rome, Italy

*Correspondence: Professor Dr MC Cornel, Clinical Genetics and EMGO Institute for Health and Care Research, VU University Medical Centre, EMGO/Clinical Genetics, BS7 D450, PO Box 7057, 1007 MB Amsterdam, The Netherlands. E-mail: mc.cornel@vumc.nl 
(HTA) and/or coordinating screening programs, many are involved in the service delivery of NBS in pediatrics, laboratory medicine and genetics. The task of EUNENBS was to supervise the work of the tender and participate in the revision of the tender deliverables, including the Expert Opinion document. The EUNENBS members have provided informally their input and advice without implying any obligation or commitment of their national authorities or organizations. Working documents were prepared reviewing most relevant scientific literature on the development of NBS policy and submitted to EUNENBS to stimulate the discussion during its meeting held on 6-7 December 2010, where the future of NBS was discussed in a workshop. Conclusions were integrated in a draft of the Expert Opinion document that was circulated by e-mail on 9 March 2011 to the membership of EUNENBS and to European Union Committee of Experts on Rare Diseases members from the Candidate and European Economic Area/European Free Trade Association countries inviting comments. This consultation ended on 6 April 2011. The preparation of the second draft, integrating the suggestions received, took place until 6 May 2011. Before the consensus meeting on 20 and 21 June 2011 in Luxembourg, the document was circulated for a second consultation, which took place from 11 to 27 May 2011, and amended considering the comments received. The Expert Opinion document was endorsed by the Boards of the International Society for Neonatal Screening and the European Society of Human Genetics in August and October 2011.

Experiences from other countries have served as useful sources, although their applicability may need to be checked against information from EU countries and agreement needs to be sought with EUNENBS. This article presents the 70 Expert Opinions, resulting from the debate among the EUNENBS members with respect to the elements that are part of a system to evaluate the quality and ethical aspects of neonatal screening in the light of available literature, as well as the proposal for a decision matrix. We furthermore provide a brief discussion.

\section{RESULTS 1: EXPERT OPINIONS}

Governance of neonatal screening

1. Screening is different from diagnostics. Screening is offered to people who either do not have or have not recognized the symptoms of the disease(s) that the screening relates to. A screening test is not intended to be diagnostic. Screening aims to identify people at sufficient risk to benefit from referral for diagnostics.

2. Haven taken notice of the fact that a European body for the HTA will be developed (European Network for Health Technology Assessment), ${ }^{5,6}$ the EUNENBS recommends a committee for neonatal screening.

3. This EU NBS committee should summarize the scientific developments (evidence, economics and ethics) ${ }^{7}$ and advice transparently. It should update relevant information at national and European level. In addition, because it will gather the widest expertise on NBS at the EU level, it should act as a central point for any stakeholder (eg, learned societies, industry and patient groups) to propose and discuss new NBS procedures.

4. The EU NBS body should promote synergies and best practice guidelines on policies concerning consent, storage of samples, pretest information for parents, etc. (benchmarking, reviewing, updating and so on).

5. The body should have a clear governance structure and accountability. It should have a role in offering advice to (national) policy makers.
6. In each country, national bodies should assess the country-specific factors, including epidemiological, economical, ethical and legal issues, and perform the monitoring and evaluation of the program.

7. A formalised decision process is needed to start the HTA of a screening and to re-evaluate the evidence for screening either periodically or on demand.

8. Actors to be involved in NBS decision making include patients' and parents' organizations, laboratory scientists, health-care workers and professional organizations, ethical, legal and economic experts, governmental and non-governmental agencies and health-care providers.

9. The role of industry, commercial parties or industrial researchers should be limited to consultation.

10. Existing examples of written policies should be translated and published, so that they could serve as examples for the countries that do not have such policies yet, but which are considering their development. The criteria used by national committees when considering new screening programs should be published. The examples of policies should cover both national and European practices in a way that could allow the assessment of trans-border issues (eg, equipment-related issues, access to relevant new technologies and appropriate screening for people moving from one country to another).

11. Systems should be in place within the EU to learn from potential generic adverse incidents that may cross national boundaries, for example, equipment-related issues.

12. Once the EU NBS body is in place and examples of good practices are available, it should be discussed to what extent harmonization of NBS in Europe is possible.

\section{Criteria to evaluate whether a screening program should be performed}

1. There is a clear need to develop and publish agreed case definitions for all disorders screened. There should be an attempt made to achieve agreement on these case definitions within the EU to facilitate assessment and international outcome studies.

2. The decision whether a screening program should be performed can be based on a framework of screening criteria updated from the traditional Wilson and Jungner criteria (W\&J), relating to disease, treatment, test and cost.

3. The interest of the child should be central in the assessment of pros and cons.

4. The European NBS body (or the national NBS bodies) should further elaborate the specifications and the operative application of the screening criteria through discussion and agreement with the EU national authorities.

5. HTA to evaluate the evidence on the effectiveness of early detection through neonatal screening and treatment should be achievable in practice. For rare conditions, best level evidence should be used. Methods need to be developed to both optimize health benefit and careful evaluation.

6. Universal screening is generally preferable to ethnical targeted screening. If there are sound reasons (eg, health gain) for targeted screening, it is important to avoid stigmatization.

7. The health system should ensure treatment to all confirmed cases diagnosed by screening. In case of suboptimal availability of treatment, it should plan to make treatment available for all confirmed cases (based on common values and principles in EU Health Systems (universality and access to good quality care)). 
8. Systems should be developed in order to support universal screening in countries where it would be beneficial, but not affordable, for economic and/or social reasons.

9. Systems should be put in place by the EU for helping the countries, where treatment is not available yet for all confirmed cases. The target of treatment for all confirmed cases should be achieved without reducing the quality of treatment.

10. The European NBS body (or the national NBS bodies) should consider other potential advantages, especially (a) avoiding a diagnostic odyssey and (b) informed reproductive choice for the next pregnancy(ies) of the parents, and later for the child, and the provision of genetic counseling to the family.

11. Screening methodology should aim to avoid unintended findings, such as cases with mild forms of the disorder screened for and information on carrier status, as much as possible.

12. If unintended results are found (such as carrier status), member states need to consider carefully how results are communicated. Parents need to be informed adequately in a way that is consistent with the individual data protection rights and the right to privacy, as well as patient rights. (Pretest information is discussed in Chapter 5 of the Expert Opinion document)(http://www.iss.it/ $\mathrm{cnmr} / \mathrm{prog} / \mathrm{cont}$.php?id=1621\&lang=1\&tipo=64.)

13. Economic evaluations of NBS programs are needed. Balancing the right to care of all patients' needs to take rare disorders into account.

14. Even if a program may be cost effective in the long run, the initial costs may represent a barrier to start. Raising specific initial funding should be considered.

15. Systems should be in place at EU level in order to support the countries, which for reasons of economic development might have difficulties in covering those initial costs.

\section{Criteria on how a screening program should be performed}

1. Before the start of an NBS program, all health-care professionals involved must be offered adequate training and sufficient participation must be achieved.

2. The provision of information needs to be organized at program management level by public health authorities and is the responsibility of the NBS program management. This should be developed in collaboration with the relevant users.

3. The information contents and communication guidelines should be defined at program management level; it may take advantage from sharing existing examples and experiences.

4. Sufficient general information on NBS should be given to prospective parents, starting during pregnancy. This could also come up in preconceptional care. Detailed information should be available upon request. On a program level, the responsibility for this pretest information needs to be clarified: public health authorities could mandate obstetric-care providers.

5. Evidence-based patient information on NBS in appropriate language should be made available on websites of the institutions responsible for the screening.

\section{Informed consent}

1. NBS must be offered to all infants in the EU.

2. It should be offered as a service governed by appropriate legal provisions, which also ensures compliance with quality requirements of other legislation (such as patient's rights, personal data protection, biobanks, research approval by ethics committee, genetic testing and genetic counseling). The health-care system should cover the costs.

3. The importance of NBS in the best interest of their child should be clarified to parents. Participation should be voluntary.

4. A specific consent should be sought for activities not strictly related to the benefit of the newborn, such as the use for research purposes.

5. The informed consent protocols should be defined at jurisdictional level, in consultation with the appropriate stakeholders; it may take advantage from sharing existing examples and experiences.

\section{Blood spot sampling}

1. Blood spot sampling between 48 and $72 \mathrm{~h}$ is preferable for most disorders in NBS programs.

2. Uptake needs to be monitored, an uptake of $100 \%$ is pursued. If informed consent is taken seriously, this value may not be reached.

3. Systems should be in place to maximize uptake and ensure that babies are not missed

4. Systems should be in place to deal with the families moving into the area and crossing national boundaries to ensure that appropriate screening has been carried out or is offered.

\section{Laboratory procedures}

1. The target values and benchmarks ensuring the quality and efficacy of laboratory procedures should be defined at program management level;

2. The development of laboratory procedures should take advantage from sharing existing examples and experiences.

3. Defined screening protocols should be published by each member state and reviewed every 1-5 years or on demand in case of recognized developments.

4. Test turnaround time within the laboratory should be kept short: for example, a maximum of $48 \mathrm{~h}$ is recommended.

\section{Blood spot storage}

1. Blood spots need to be stored for quality control in the NBS screening laboratory for at least 5 years.

2. Blood spot storage should ensure appropriate protection of sensitive personal information and of biological samples (eg, compliance with the relevant regulations).

3. Informed consent should be asked, at least for activities not strictly related to the benefit of the newborn, such as storage for quality control and research. For use of the blood spot after 18 years, the child should have the possibility to consent or dissent.

4. Use of blood spots for research purposes is subject to national specific ethical regulations (eg, definition of research objectives and timing, informed consent and approval by the ethical committee). The potential interest for research and the possible misuse of residual NBS specimens have increased the need for regulation of specimen storage and access policies at the European level for both ethical and legal reasons. At the European level, major differences in regulations should be avoided in view of trans-border health care and international research. 


\section{Communication of positive result}

1. Communication of the need for additional clinical investigations should be preferably carried out by specialists. In case good information has been provided to parents before the sampling/ birth, this communication may be carried out also by non-experts, if clearly instructed what to communicate.

2. The information contents and communication guidelines, for the communication of the need for additional clinical investigations to parents, should be defined at program management level and published; there may be advantages to sharing existing examples and experiences.

3. For every positive NBS result a diagnostic confirmation test, performed by established laboratory methods according to predefined standards, must take place, for most disorders within $24 \mathrm{~h}$ or the next working day after communicating a positive screening result.

4. Communication means should ensure timely delivery to parents, with check on receipt and understanding. Communication of any result, including negative results, may contribute to quality control and parental well-being.

\section{Confirmation of diagnosis and treatment}

1. Defined 'diagnostic protocols' should be developed, which relate directly to the case definition. Protocols on whom to treat as patient, including referral to clinical services, should be available at program level.

2. Protocols for confirmation of diagnosis and guidelines for treatment should be defined at program management level; there may be advantages to sharing existing examples and experiences.

3. Communication after a confirmed diagnosis is extremely important. Personal communication by physicians can be supported by information from accredited webportals.

\section{Communication of unintended findings}

1. Parents should be given the possibility to be informed of any unintended finding that could be relevant, to the extent this is consistent with laws, individual data protection rights and the right to privacy.

2. Different positions have been taken in the debate on unintended findings. Discussion is needed in countries to develop policy and legislation, if appropriate. This should be published.

3. As far as unintended but relevant information for the health of the child or mother is concerned, parents should be given the possibility to be informed. For the return of information on carrier status, a separate decision, consistent with other relevant national health regulations, is needed in each country. This is because carrier information is mainly important for reproductive choice of the parents and not directly for the health of the screened newborn. The content of the information and guidelines for its communication to parents should be defined at program management level; it may take advantage from sharing existing examples and experiences.

\section{Quality assurance of laboratory results}

1. NBS laboratories should be certified and participate in external quality assurance/control programs. The EU NBS committee should advise on EQA and poor performance, and offer educational support to poorly performing laboratories.

2. Within a jurisdiction the number of laboratories should be limited. Optimal quality performance and cost effectiveness requires a minimum number of samples handled, such as 30000-50000 samples per year.

\section{Screening program evaluation}

1. The quality of the process of the program needs to be monitored regularly (possibly annually) to allow the identification of steps requiring improvement and the adoption of appropriate corrective measures. Results should be made available by open access.

2. Evaluation of specific aspects of NBS programs must be considered for aspects other than those regularly monitored, such as recently changed information policies.

3. Databases are needed to monitor and evaluate the program. As all NBS conditions are rare, international collaboration may help to facilitate evaluation.

4. Systems should be in place to ensure that feedback of confirmed diagnosis and long-term outcomes are available for program evaluation, also in case of screened children moving abroad.

\section{Epidemiological evaluation}

1. Collaborative international projects are needed to assess the longterm follow-up of the patients with rare conditions identified in NBS programs. Both evaluation of programs (expert opinion no. 65), and the success of screening and treatment for patients and families are needed. The EU should take a pro-active approach to organize long-term follow-up.

Features of disorders, which might be considered in the gradual expansion of NBS in EU

1. Training on all aspects of improving NBS programs should be facilitated at EU level.

2. EU countries should consider the assessment of the first group of disorders (Chapter 5 of the Expert Opinion document) (http:// www.iss.it $/ \mathrm{cnmr} / \mathrm{prog} / \mathrm{cont}$.php?id=1621\&lang=1\&tipo=64) on the basis of local/national conditions in case that they intend to expand their NBS. This process and conclusions should be published.

3. The EU NBS body, charged with the assessment of the evidence and possibilities for neonatal screening, ${ }^{8}$ might consider initiating its activity with reviewing the evidence for disorders to be screened. For the first group of disorders, several countries have assessed the evidence already. Especially the conditions in the second group (Chapter 5 of the Expert Opinion document) (http://www.iss.it/cnmr/prog/cont.php?id=1621\&lang=1\&tipo=64), where limited evidence is available or different conclusions were reached need to be prioritized.

4. There is an opportunity to use the moment of blood spot screening for other screening programs concerning, for example, hearing loss, hips, eyes and heart. 


\section{RESULT 2: PROPOSED MODEL OF DECISION-MAKING MATRIX}

(1) Does your country or health-care jurisdiction have a neonatal screening program?

(a) If no: start neonatal screening for congenital hypothyroidism (the reason for the choice of congenital hypothyroidism is twofold: (1) congenital hypothyroidism is (one of) the most prevalent congenital disorders, the prevalence being largely independent of ethnicity; (2) the screening and confirmatory methodology is relatively simple. All European countries that contributed to the current Practices Document (http://www. iss.it/cnmr/prog/cont.php?id=1621\&lang=1\&tipo=64; refs 3,4) screen for congenital hypothyroidism.).

(2) If YES, consider disorders for which a neonatal screening program exists elsewhere, or for which research shows promising results. For each disorder:

(a) Can, according to international experience, considerable, irreparable damage be prevented by neonatal screening or other benefits for the patient and the family be achieved? Assessment includes:

(i) The condition sought should be an important health problem (W\&J1). ${ }^{1}$

(ii) There should be an accepted benefit for patients with recognized disease (W\&J2).

(iii) There should be a recognizable latent or early symptomatic stage (W\&J4).

(iv) The natural history of the condition, including development from latent to declared disease, should be adequately understood (W\&J7).

(b) Is, according to international experience, a good test available? (Sensitivity, specificity, positive predictive value and acceptability) Assessment includes:

(i) There should be a suitable test or examination (W\&J5).

(ii) The test should be acceptable to the population (W\&J6).

(iii) There should be an agreed policy on whom to treat as patients (W\&J8).

(3) If both questions YES, consider desirability in your country/ region:

(a) Is the disorder an important health problem in your country?

(b) Is the test acceptable for the population from cultural/ethical perspective (unintentional findings; carrier status; mild and late-onset forms).

(4) If the previous questions are answered YES, consider the feasibility:

(a) Compare the burden of the disorders for the health system with the cost of screening, with a view to ensuring equity of access to health care and considering other feasible options.

(i) The cost of case finding (including diagnosis and treatment of patients diagnosed) should be economically balanced in relation to possible expenditure on medical care as a whole (W\&J9).

(ii) What is the birth prevalence of the disorder(s)? (b) Can facilities be made available for adequate surveillance, prevention, treatment, education, counseling and social support? Assessment includes:

(i) Facilities for diagnosis and treatment should be available (W\&J3).

(ii) Case finding should be a continuing process and not a 'once and for all' project (W\&J10).

(iii) Is a good test available in your country?

(iv) Are sufficient diagnostic specialists available?

(v) Is treatment available in your country?

(vi) Are sufficient treatment specialists available?

(vii) Are there patients' associations which may provide support to the patient and/or the family?

(5) If NBS is considered desirable and feasible, take care of adequate quality of the program, including:

(a) Training of relevant health-care providers.

(b) Information to prospective parents.

(c) Informed consent, both general and specific, on communication of carrier status information and sample storage for research use.

(d) Procedures for blood spot sampling, laboratory handling and storage of cards.

(e) Protocols for communication of health-care providers in case of positive results.

\section{DISCUSSION}

Experts from a diversity of backgrounds in health policy making, coordinating screening programs, laboratory and clinic engaged in a series of meetings in 2010 and 2011 to analyze the diversity of NBS practices in EU and other countries and to develop Expert Opinions on NBS policy. NBS programmes are very diverse in these countries. Given the fast technological changes and the increasing possibilities to treat or even cure rare diseases diagnosed after NBS, the importance of developing a reference framework for NBS practices and policy making became clear.

Not only in Europe, also in the USA and other continents NBS is under revision. Horizon scanning, quality control, epidemiological follow-up and other activities may profit from international collaboration, for instance with the USA Secretary's Advisory Committee on Heritable Disorders in Newborns and Children (http://www. thefederalregister.com/d.p/2010-04-26-2010-9625). For the followup, it is of great importance that case definitions are the same world-wide (expert opinion no. 13). The International Society for Neonatal Screening could have an important role here.

The analyses and opinions agreed by the EUNENBS experts within this tender have been presented to the European Union Committee of Experts on Rare Diseases. This advisory committee for EU policy on rare diseases is now debating on collaborative solutions to further improve their NBS programs and fill the gaps highlighted in the tender documents without impacting on their national prerogatives in the delivery of care.

Beside the initiatives that will be taken at EU level, the analyses and opinions produced by the tender activities may guide further national policy developments, bilateral and international initiatives.

\section{ACKNOWLEDGEMENTS}

This work is funded by the EU with a grant of Euro 399755 (contract number 20096206 of the Executive Agency for Health and Consumers). The opinions 
expressed in this document are those of the contractor only and do not represent the official position of the Executive Agency for Health and

Consumers. We are gratefully indebted to the members of EUNENBS.

1 Wilson JMG, Jungner G: Principles and Practice of Screening for Disease. Geneva: WHO, 1968. Available from: http://whqlibdoc.who.int/php/WHO_PHP_34.pdf

2 Council Recommendation of 8 June 2009 on an action in the field of rare diseases. Available from: http://eur-lex.europa.eu/LexUriServ/LexUriServ.do?uri=OJ:C:2009:151: 0007:0010:EN:PDF

3 Loeber JG, Burgard B, Cornel MC et al: Newborn screening programmes in Europe; arguments and efforts regarding harmonization. Part 1 - from blood spot to screening result. J Inherit Metab Dis 2012; 35: 603-611.

4 Burgard P, Rupp K, Lindner $\mathrm{M}$ et al: Newborn screening programmes in Europe; arguments and efforts regarding harmonization. Part 2 - from screening laboratory results to treatment, follow-up and quality assurance. J Inherit Metab Dis 2012; 35: 613-625.

5 Kristensen FB, Mäkelä M, Neikter SA et al: European network for Health Technology Assessment (EUnetHTA). European network for health technology assessment,
EUnetHTA: planning, development, and implementation of a sustainable European network for health technology assessment. Int J Technol Assess Health Care 2009; 25:Suppl 2 107-116.

6 EUnetHTA Joint Action - new phase in EUnetHTA development. Available from: http:// www.eunethta.eu/Public/Home/

7 Grosse SD, Rogowski WH, Ross LF, Cornel MC, Dondorp WJ, Khoury MJ: Population screening for genetic disorders in the 21st century: evidence, economics, and ethics. Public Health Genomics 2010; 13: 106-115.

8 Calonge N, Green NS, Rinaldo P et al: Advisory Committee on Heritable Disorders in Newborns and Children. Committee report: method for evaluating conditions nominated for population-based screening of newborns and children. Genet Med 2010; 12: 153-159.

cc) (i) () $\odot$ This work is licensed under a Creative Commons Attribution-NonCommercial-NoDerivs 3.0 Unported License. To view a copy of this license, visit http://creativecommons. org/licenses/by-nc-nd/3.0/ 